\title{
Cognitive and Behavioral Disorders in Patients with Precuneal Infarcts
}

\author{
Emre Kumral $^{\mathrm{a}}$ Fatma Ece Bayam $^{\mathrm{b}}$ Hüseyin Nezih Özdemir ${ }^{\mathrm{a}}$ \\ aNeurology Department, Ege University Medical School Hospital, İzmir, Turkey; ${ }^{\text {} N e u r o l o g y ~ D e p a r t m e n t, ~ A c ı b a d e m ~}$ \\ Hospital, Bursa, Turkey
}

\section{Keywords}

Precuneal cortex $\cdot$ Ischemic stroke $\cdot$ Pericallosal artery ·

Memory $\cdot$ Emotion

\begin{abstract}
Objective: Ischemic stroke of the precuneal cortex (PC) alone is extremely rare. This study aimed to evaluate the clinical, neurocognitive, and behavioral characteristics of isolated PC infarcts. Methods: We assessed neuropsychological and behavioral findings in 12 patients with isolated PC infarct among 3,800 patients with ischemic stroke. To determine the most frequently affected brain locus in patients, we first overlapped the ischemic area of patients with specific cognitive disorders and patients without specific cognitive disorders. Second, we compared both overlap maps using the "subtraction plot" function of MRIcroGL. Results: Patients showed various types of cognitive disorders. All patients experienced more than 1 category of cognitive disorder, except for 2 patients with only 1 cognitive disorder. Lesion topographical analysis showed that damage within the anterior precuneal region might lead to consciousness disorders $(25 \%)$, self-processing impairment (42\%), visuospatial disorders (58\%), and lesions in the posterior precuneal region caused episodic and semantic memory impairment (33\%).
\end{abstract}

The whole precuneus is involved in at least one body awareness disorder. The cause of stroke was cardioembolism in 5 patients (42\%), large artery disease in 3 (25\%), and unknown in 4 (33\%). Conclusions: This study showed a wide variety of neuropsychological and behavioral disorders in patients with precuneal infarct. Future studies are needed to achieve a proper definition of the function of the precuneus in relation to the extended cortical areas. PC region infarcts have been found to predict a source of embolism from the large arteries or heart.

(c) 2021 S. Karger AG, Basel

\section{Introduction}

The precuneus is a part of the superior parietal lobule (SPL) on the medial surface of each brain hemisphere, trapped between the occipital cortex and the paracentral lobe and covered by the sagittal sinus and bridge vessels. Precuneus receives blood from the precuneal artery, a branch of the caudal branch of the pericallosal artery originating from the anterior cerebral artery.

As a relational area, the precuneal cortex (PC) is responsible for some aspects of multimodal integration, receiving information from different sensory modalities karger@karger.com www.karger.com/ene
(C) 2021 S. Karger AG, Basel

Karger'
Correspondence to:

Emre Kumral, emre.kumral@ege.edu.tr 
which are combined to provide harmonious perceptual experience. The integration within precuneus effectuates a central role for a large spectrum of highly integrated functions, including visuospatial imagery, episodic memory, self-processing operations, and body awareness in general, especially the integration of tactile, proprioceptive, vestibular, and visual inputs [1-7]. The current information about the anatomy of precuneus shows that it is a versatile functional area for behavioral correlates, with broad connectivity to both subcortical and cortical structures, as demonstrated by connectivity and neuroimaging findings $[4,7]$.

The precuneus has traditionally received little study and has been debated much less, since it is rarely damaged in stroke, slow-growing tumors, and trauma; however, its critical location and widespread connection demonstrate that precuneus is a strategic area that can perform various cognitive and behavioral functions [8-10]. There are no systematic neurocognitive studies on the results of isolated ischemic lesions of the PC. We describe here a series of patients who presented an ischemic lesion damaging the $\mathrm{PC}$ associated with specific clinical pictures and assess its role in the higher order cognitive functions and conscious state.

\section{Subjects and Methods}

Between January 2013 and October 2019, 12 patients with ischemic lesions restricted to the precuneus were recruited from 3,800 patients with ischemic stroke who were admitted to our Stroke Unit [11]. In patients with previous stroke, old lesions on neuroimaging, or concomitant acute multiple lesions outside the precuneus were excluded. The diagnostic criteria of our database have been extensively detailed elsewhere [11].

All patients had a standardized evaluation for their clinical and neuropsychological pictures, and consecutively recorded variables included age, gender, stroke risk factors, blood pressure, etiological subtypes, and topography of lesions on CT and MRI. CT and magnetic resonance, $2 \mathrm{D}$-echocardiography, and 24-h electrocardiography (Holter) monitoring were performed in all cases. The etiology of stroke was presumed according to the criteria described previously [12]. This research was reviewed and approved by the Ethics Committee of Ege University Medical Center (2010/111), and a written informed consent was obtained from all patients.

\section{Neuropsychological Assessment}

Based on the current knowledge about the precuneus, different aspects of neuropsychological syndromes were systematically evaluated in all patients. The neurocognitive assessment was performed by 2 neuropsychologists with accreditation in neurocognitive science in the first week of stroke and 3 months after stroke, as part of routine assessment. A z-score of each patients' neurocognitive test point was calculated and a score below the cutoff or a $\mathrm{z}$-score inferior or equal to -1.65 was considered as pathological.
Different aspects of cognitive functions were analyzed on the basis of similarity to the tasks handled and cross-reference sets and arbitrarily classified into 7 broad domains [7]: (1) orientation assessed by asking the patient's orientation in space, time, and person; (2) self-processing included self-referential judgments evaluated by asking subjects to discriminate his/her own face from the faces of strangers, sense of self agency proved whether the patient was aware of feeling of control over volitional actions and their consequences, and social cognition evaluated by asking the patient to interpret other people's feelings and emotions; (3) executive functions assessed by Trail Making Test (the part B minus the part A) and the Stroop Test (the interference part); (4) memory functions included autobiographical memory assessed by asking the patients to report the most important personal events of their life or to report self-defined memory; episodic memory assessed by Rey Auditory Verbal Learning Test (RAVLT), including measures of immediate free recall/new learning (list A trials 1-3; list B); semantic memory assessed by category fluency task (animals); (5) visuospatial dysfunctions such as visuospatial disorientation evaluated by Benton's Judgment of Line Orientation Test; spatial neglect assessed with Line bisection task; motor neglect (underutilization of the upper extremity for tasks); visual extinction (when bilateral visual stimulations were repeatedly judged as unilateral stimulation); optic ataxia (disturbance to reach and grasp the examiner's finger placed successively in different sectors of the visual field); visual agnosia assessed by a subtest "incomplete letters" of the Visual Object and Space Perception Battery; (6) body awareness assessment included, anosognosia for hemiplegia, fading limb, autotopagnosia for body parts or for body sensations, motor neglect, micro- or macrosomatognosia, supernumerary limb, tactile allochiria, finger agnosia, alien hand syndrome, hemiconcern syndrome, and tactile extinction; (7) other neurological/neuropsychological syndromes were systematically checked such as constructional apraxia, ideational apraxia, dressing apraxia, optic apraxia, verbal fluency, and semantic matching assessed by Pyramids and Palm Trees test (PPTT), and semantic fluency evaluated with the semantic fluency task. Tables 2 and 3 presented neuropsychological and behavioral findings.

\section{Magnetic Resonance Imaging and Data Acquisition, and Normalization}

MRI was performed within 1 week after stroke and 3 months after stroke by 3T scanner (Siemens Sonata, Siemens Medical Solutions, Erlangen, Germany) as part of the standard examination. Routine MRI sequences are obtained, including T1-weighted imaging, T2-weighted imaging, DWI, and fluid-attenuated inversion recovery. Two vascular neurologists with accreditation in neuroimaging retrospectively reviewed the noncontrast MRI sequences blinded to clinical variables. For each normalized individual imaging, we used SPM12 (https://www.fil.ion.ucl.ac.uk/spm/software/) with standard parameters applied in the MATLAB environment (2019 version, MathWorks). During this step, all normalized MRIs (MNI152 brain) were manually controlled to verify that all normalizations were of adequate quality. Then, we manually drawn the ischemic areas and converted them into binary images using MRIcroGL software (http://www.mccauslandcenter.sc.edu/mricrogl/home). Ischemic lesion territories were subdivided according to their anatomic location within the PC, such as antero-dorsal precuneal territory, postero-dorsal precuneal territory, and whole precuneal territory. 


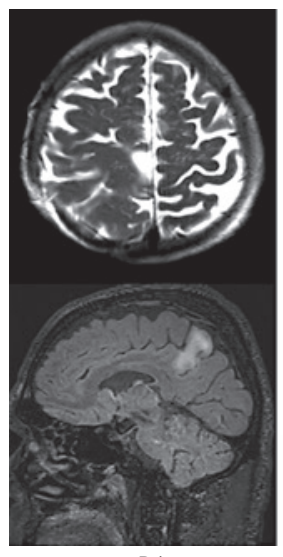

P1

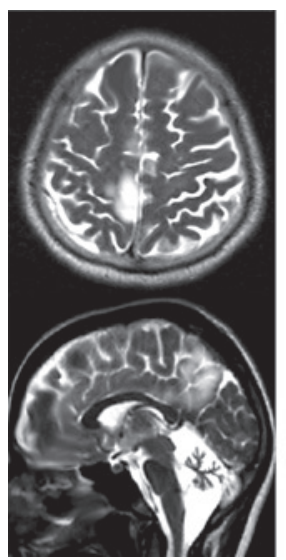

P7

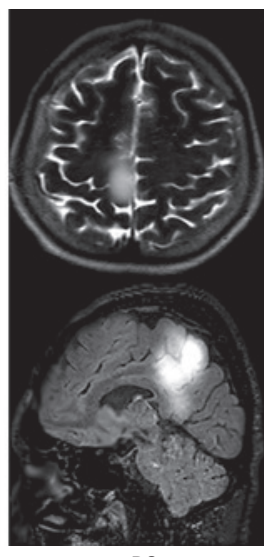

P2

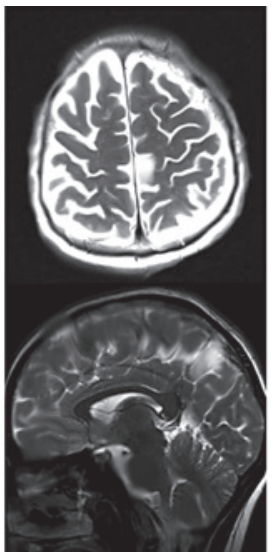

P8

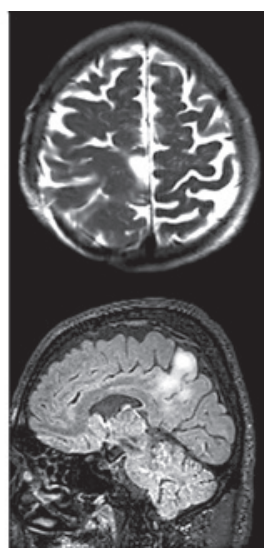

P3

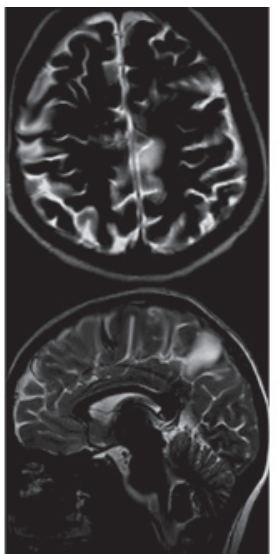

P9

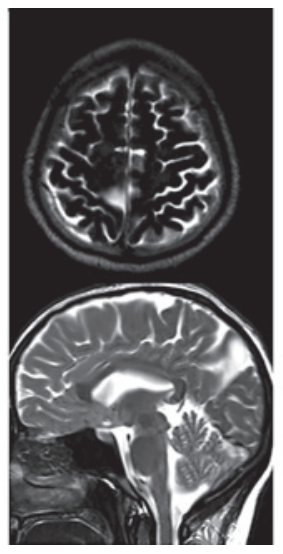

P4

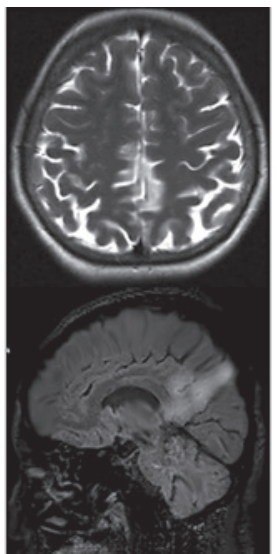

P10

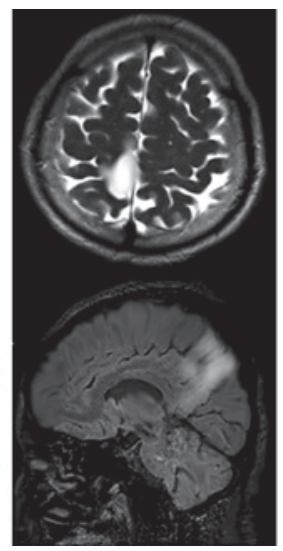

P5

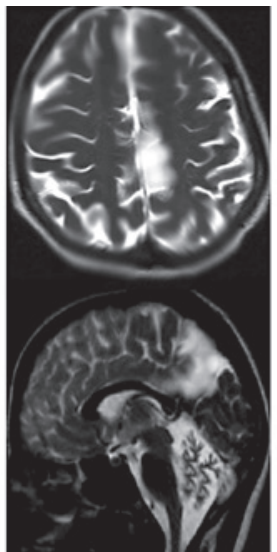

P11

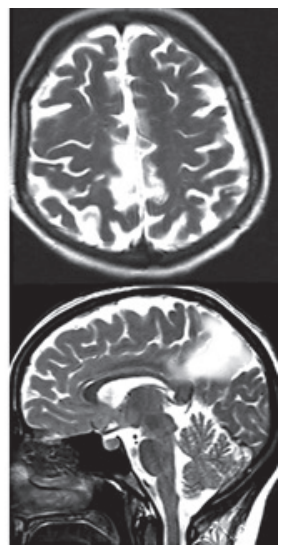

P6

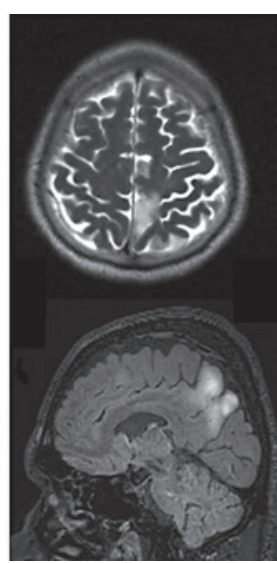

P12

Fig. 1. Axial and sagittal MRI images of the brain showing high intensity in the anterior and posterior parts of the PC. Posterior cingulate gyrus was involved partially in 5 patients (P2, P5, P6, P10, and P11) and SPL in 4 patients (P2, P5, P7, and P11). SPL, superior parietal lobule; PC, precuneal cortex.

\section{Subtraction Plots}

To determine which precuneal areas are most frequently affected by ischemic lesions in patients with self-processing, memory, visuospatial, body awareness, and semantic disorders, we first overlapped ischemic lesions of patients with and without these disorders. Then, we compared both overlap maps using MRIcroGL's "subtraction plot" function. For this analysis, ischemic lesions on the left side $(n=5)$ were inverted.

\section{Results}

The patient population consisted of 5 females and 7 males, with a mean \pm standard deviation (SD) age of 40.07 \pm 11.08 years $(22 \pm 55)$ and a mean educational level of 13 \pm 2.55 (SD) years (9-18). All patients were right-handed, and for 7 of them, the ischemic lesion was located in the right hemisphere (Fig. 1a). Demographic and clinical features are given in Table 1.

In the first day of stroke, there were miscellaneous neurological and behavioral complaints such as mild transitory hemiparesis in 3 patients ( $\mathrm{P} 2, \mathrm{P} 6$, and $\mathrm{P} 11)$, hypokinesia in 3 (P3, P5, and P12), illusory feelings of movement in 1 (P5), feelings of strangeness in 2 (P5 and $\mathrm{P} 11)$, topographic disorientation in 5 (P1, P3, P2, P6, and $\mathrm{P} 12)$, anosognosia for hemiparesis (P2, P6, and P11), somatoparaphrenia in 2 ( $\mathrm{P} 1$ and $\mathrm{P} 2$ ), and left/right indistinction only in 1 patient (patient P12) (Fig. 2) (Tables 2, $3)$.

\section{Consciousness Disorders}

At the consciousness level, 3 patients (P2, P6, and P11) had transitory confusion at stroke onset. These patients 
Table 1. Demographic and clinical findings

\begin{tabular}{|c|c|c|c|c|c|c|c|c|c|c|c|c|c|c|}
\hline Age, years & 60 & 57 & 64 & 70 & 66 & 77 & 76 & 58 & 68 & 67 & 72 & 79 & $67.83 \pm 7.33^{*}$ & - \\
\hline Infarct volume, $\mathrm{cm}^{3}$ & 10.5 & 41.2 & 18.5 & 13.8 & 34.6 & 38.4 & 22.8 & 14.2 & 13.6 & 30.4 & 38.1 & 28.6 & $25.39 \pm 11.18^{*}$ & - \\
\hline Sex & M & M & $\mathrm{F}$ & M & $\mathrm{F}$ & M & M & M & M & $\mathrm{F}$ & $\mathrm{F}$ & $\mathrm{F}$ & - & $7 \mathrm{M}(58) / 5 \mathrm{~F}(42)$ \\
\hline Infarct location & $\mathrm{AD}$ & $\mathrm{AD}$ & $\mathrm{AD}$ & $\mathrm{PD}$ & $\mathrm{C}$ & $\mathrm{C}$ & $\mathrm{C}$ & $\mathrm{AD}$ & $\mathrm{AD}$ & $\mathrm{PD}$ & $\mathrm{C}$ & $\mathrm{C}$ & - & $\begin{array}{l}5 \mathrm{AD} /(42) \\
2 \mathrm{PD} /(17) \\
5 \mathrm{C} /(42)\end{array}$ \\
\hline Manual laterality & Right & Right & Right & Right & Right & Right & Right & Right & Right & Right & Right & Right & - & $8 \mathrm{R} /(100)$ \\
\hline
\end{tabular}

$\mathrm{AD}$, antero-dorsal precuneus; $\mathrm{PD}$, postero-dorsal precuneus; $\mathrm{C}$, complete involvement; LAD, large artery disease (extracranial or intracranial atherosclerosis causing $\geq 50 \%$ luminal stenosis); $\mathrm{CE}$, cardioembolism; UN, unknown; M, male; F, female; R, right; L, left. Values in parentheses are percentage of raws. * Mean $\pm \mathrm{SD}$.

Table 2. Clinical findings of patients with precuneal infarct and frequency of findings at first week after stroke and 3 months after stroke

\begin{tabular}{|c|c|c|c|c|c|c|c|c|c|c|c|c|c|}
\hline Clinical findings & $\begin{array}{l}\mathrm{P} 1 \\
\mathrm{SD} / 3 \mathrm{M}\end{array}$ & $\begin{array}{l}\mathrm{P} 2 \\
\mathrm{SD} / 3 \mathrm{M}\end{array}$ & $\begin{array}{l}\mathrm{P} 3 \\
\mathrm{SD} / 3 \mathrm{M}\end{array}$ & $\begin{array}{l}\mathrm{P} 4 \\
\mathrm{SD} / 3 \mathrm{M}\end{array}$ & $\begin{array}{l}\mathrm{P} 5 \\
\mathrm{SD} / 3 \mathrm{M}\end{array}$ & $\begin{array}{l}\mathrm{P} 6 \\
\mathrm{SD} / 3 \mathrm{M}\end{array}$ & $\begin{array}{l}\mathrm{P} 7 \\
\mathrm{SD} / 3 \mathrm{M}\end{array}$ & $\begin{array}{l}\mathrm{P} 8 \\
\mathrm{SD} / 3 \mathrm{M}\end{array}$ & $\begin{array}{l}\mathrm{P} 9 \\
\mathrm{SD} / 3 \mathrm{M}\end{array}$ & $\begin{array}{l}\mathrm{P} 10 \\
\mathrm{SD} / 3 \mathrm{M}\end{array}$ & $\begin{array}{l}\mathrm{P} 11 \\
\mathrm{SD} / 3 \mathrm{M}\end{array}$ & $\begin{array}{l}\mathrm{P} 12 \\
\mathrm{SD} / 3 \mathrm{M}\end{array}$ & $\begin{array}{l}\text { Frequency, \% } \\
\text { SD/3M }\end{array}$ \\
\hline Confusion & $-1-$ & $+1-$ & $-1-$ & $-1-$ & $-1-$ & $+/-$ & $-1-$ & $-1-$ & $-1-$ & $-1-$ & $+/-$ & $-1-$ & $25 / 0$ \\
\hline Vestibular findings/dizziness & $-1-$ & $-1-$ & $-1-$ & $-1-$ & $-1-$ & $-1-$ & $-1-$ & $-1-$ & $-1-$ & $-1-$ & $+/-$ & $-1-$ & $8 / 0$ \\
\hline Hypokinesia and bradykinesia & $-1-$ & $-1-$ & $+/-$ & $-1-$ & $+/-$ & $+/-$ & $-1-$ & $-1-$ & $-1-$ & $-1-$ & $-1-$ & $+/-$ & $33 / 0$ \\
\hline Illusory feelings of movement & $-1-$ & $-1-$ & $-1-$ & $-1-$ & $+/-$ & $-1-$ & $-1-$ & $+/-$ & $-1-$ & $-1-$ & $-1-$ & $-1-$ & $8 / 0$ \\
\hline Feelings of strangeness & $-1-$ & $+1-$ & $-1-$ & $-1-$ & $-1-$ & $-1-$ & $-1-$ & $-1-$ & $+/-$ & $-1-$ & $-1-$ & $-1-$ & $17 / 0$ \\
\hline Topographic disorientation & $+1-$ & $+1-$ & $+1-$ & $-1-$ & $-1-$ & $+/-$ & $-1-$ & $-1-$ & $-1-$ & $-1-$ & $-1-$ & $+/-$ & $42 / 0$ \\
\hline Anosognosia for hemiparesis & $-1-$ & $+/+$ & $-1-$ & $-1-$ & $-1-$ & $+/-$ & $-1-$ & $-1-$ & $-1-$ & $-1-$ & $+/-$ & $-1-$ & $25 / 8$ \\
\hline Somatoparaphrenia & $+/-$ & $+/-$ & $-1-$ & $-1-$ & $-1-$ & $-1-$ & $-1-$ & $-1-$ & $-1-$ & $-1-$ & $-1-$ & $-1-$ & $17 / 0$ \\
\hline Left/right indistinction & $-1-$ & $-1-$ & $-1-$ & $-1-$ & $-1-$ & $-1-$ & $-1-$ & $-1-$ & $-1-$ & $-1-$ & $-1-$ & $+/-$ & $8 / 0$ \\
\hline Finger agnosia & $-1-$ & $+/-$ & $-/-$ & $-/-$ & $-1-$ & $-1-$ & $-1-$ & $-1-$ & $-1-$ & $-1-$ & $+1-$ & $-1-$ & $17 / 0$ \\
\hline
\end{tabular}

P2, P6, and P11 are the sole patients for whom a hemiparesis was observed at the onset of stroke. SD, first week after stroke; 3M, 3 months after stroke.

had time disorientation and attention deficit to contralateral side of the lesion.

\section{Self-Processing Disorders}

These impairments were found in the first week of stroke. One patient (P2) experienced self-referential judgment disorder characterized by inability to discriminate his own face from the faces of strangers. This finding disappeared in 1 day after stroke. Three patients (P4, P5, and P11) presented loss of sense of agency who were not aware of whether they were doing volitional hand movements. Two patients (P4 and P12) could not interpret the feelings and thoughts of others and expressed that it does not feel other selves in the short questionnary for social cognition. Patients with self-processing disorders (SPDs; $n=5$ ) were more often damaged at the antero-dorsal precuneus level (maximum overlap of $80 \%$ ). On the other hand, other patients $(n=7)$ were more frequently affected by the postero-dorsal precuneus (maximum overlap of 42\%) (Fig. 3a). 

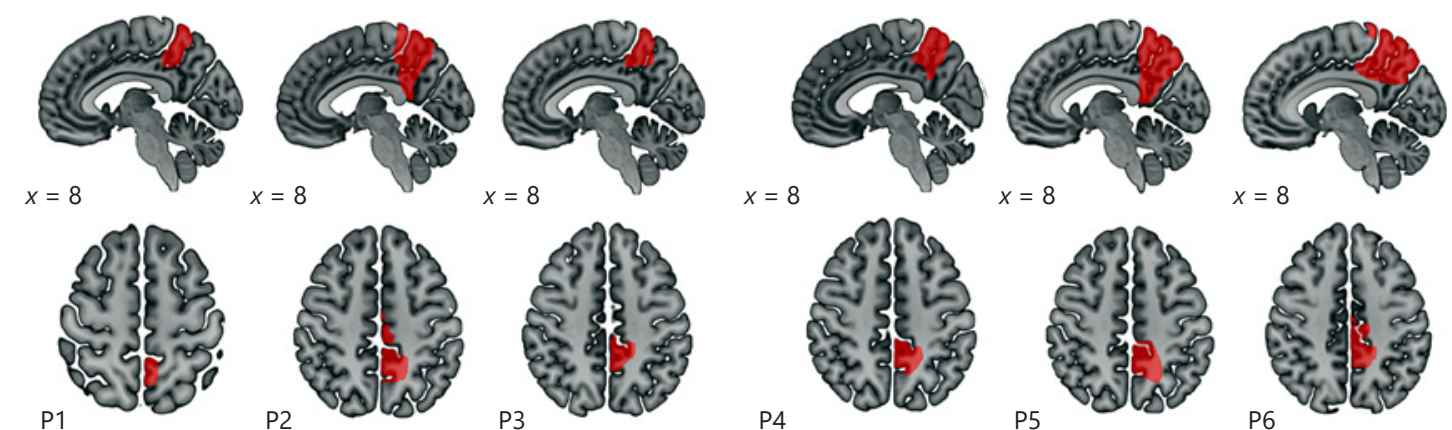

$x=8$

P3
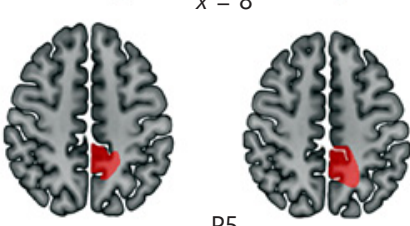

$x=8$

P4

P5
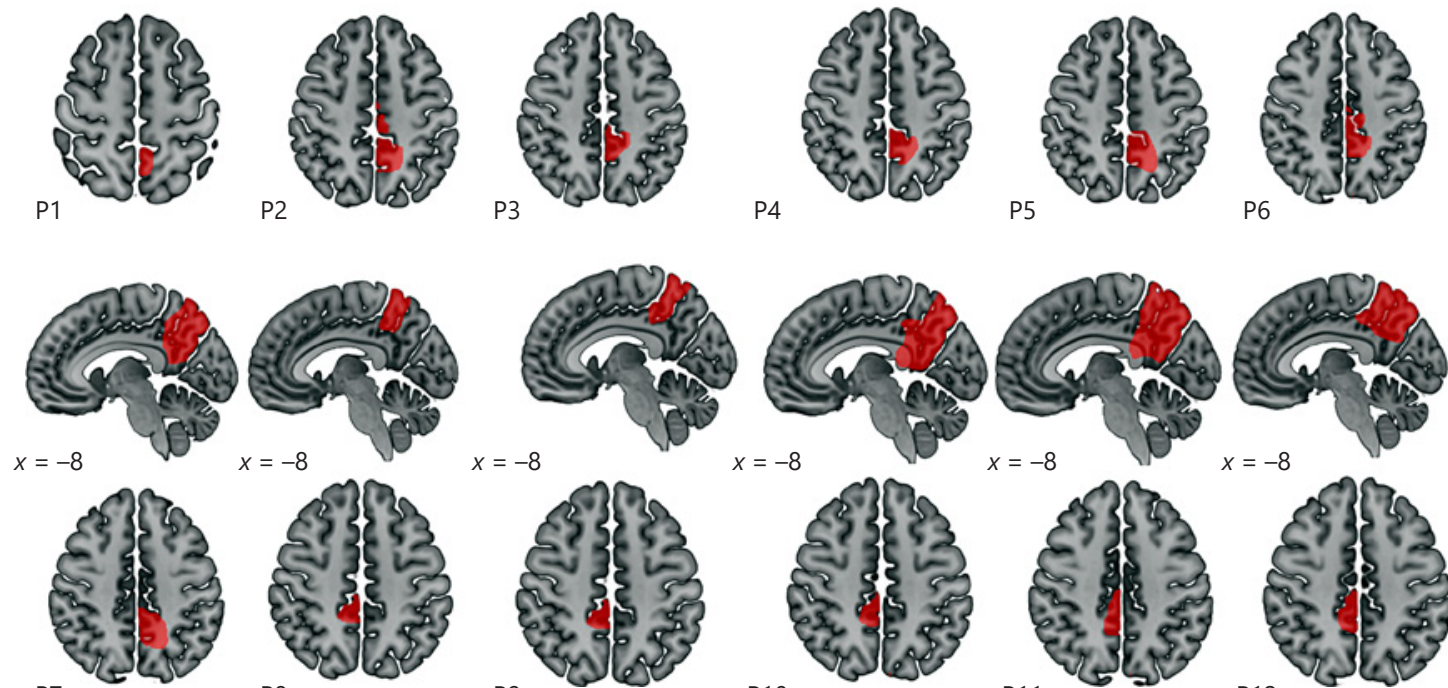

a $\mathrm{P7}$

P8

P9
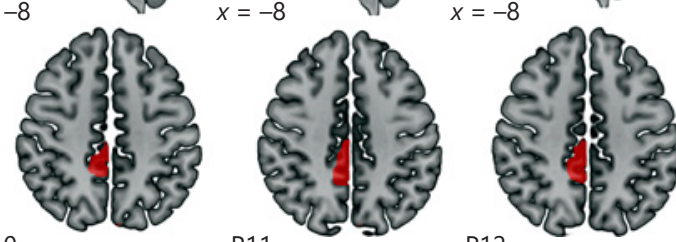

P12

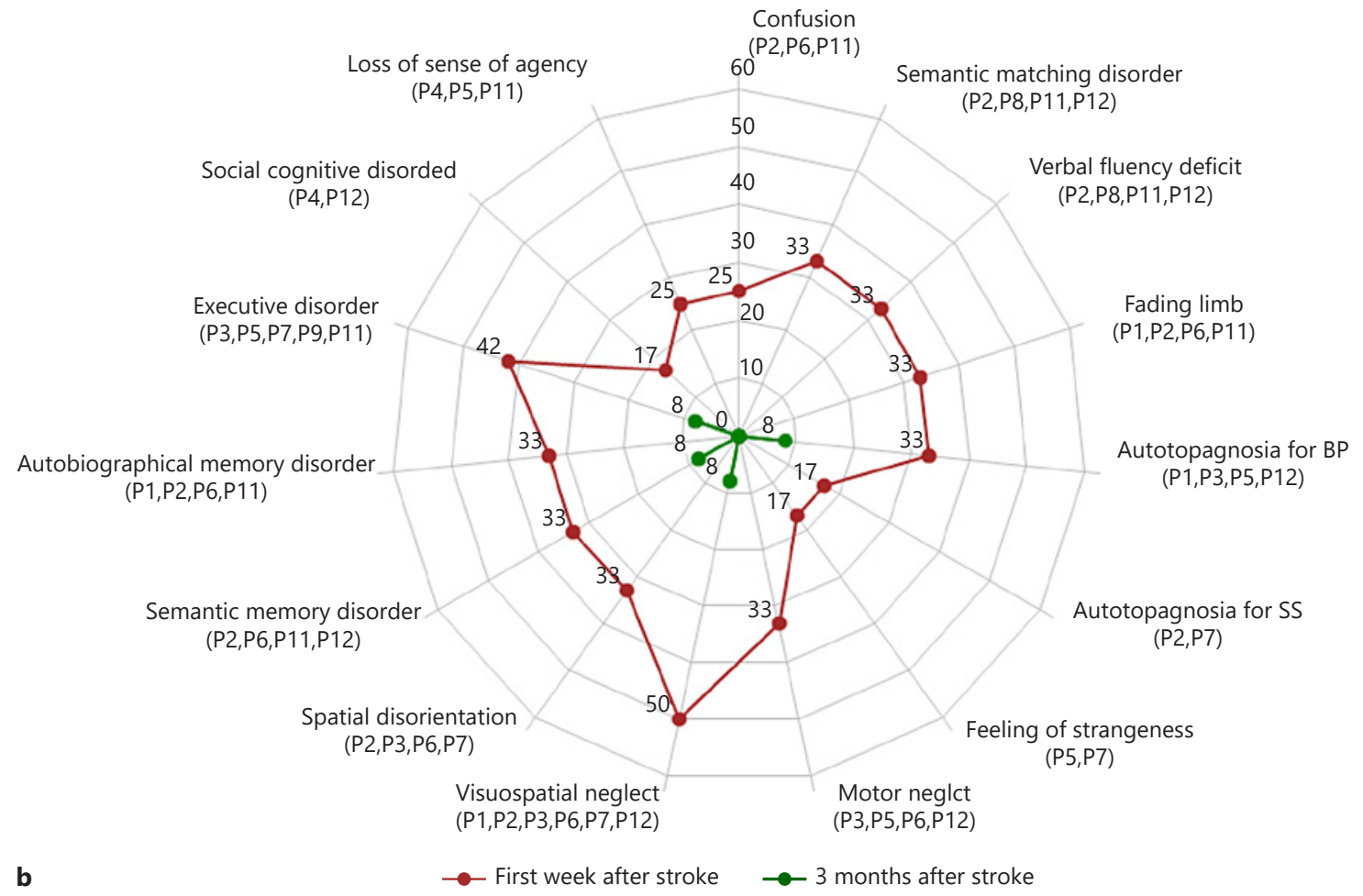

Fig. 2. Schematic drawings of ischemic lesions. a Ischemic damaged sectors of the precuneus are plotted individually onto the standard MNI152 template using MRIcroGL (https://www.mccauslandcenter.sc.edu/mricrogl/ home). b Radar plot of cognitive deficits observed in the first week after stroke, and 3 months after stroke. BP, autotopagnosia for body parts; SS, autotopagnosia for body sensations (see also Tables 2, 3). 
Table 3. Neuropsychological disorders of patients with precuneal infarct at first week after stroke and 3 months after stroke

\begin{tabular}{|c|c|c|c|c|c|c|c|c|c|c|c|c|c|}
\hline Irocognitive characteristics & $\begin{array}{l}\mathrm{P} 1 \\
\mathrm{SD} / 3 \mathrm{M}\end{array}$ & $\begin{array}{l}\mathrm{P} 2 \\
\mathrm{SD} / 3 \mathrm{M}\end{array}$ & $\begin{array}{l}\mathrm{P} 3 \\
\mathrm{SD} / 3 \mathrm{M}\end{array}$ & $\begin{array}{l}\mathrm{P} 4 \\
\mathrm{SD} / 3 \mathrm{M}\end{array}$ & $\begin{array}{l}\mathrm{P} 5 \\
\mathrm{SD} / 3 \mathrm{M}\end{array}$ & $\begin{array}{l}\mathrm{P} 6 \\
\mathrm{SD} / 3 \mathrm{M}\end{array}$ & $\begin{array}{l}\mathrm{P} 7 \\
\mathrm{SD} / 3 \mathrm{M}\end{array}$ & $\begin{array}{l}\mathrm{P} 8 \\
\mathrm{SD} / 3 \mathrm{M}\end{array}$ & $\begin{array}{l}\mathrm{P} 9 \\
\mathrm{SD} / 3 \mathrm{M}\end{array}$ & $\begin{array}{l}\mathrm{P} 10 \\
\mathrm{SD} / 3 \mathrm{M}\end{array}$ & $\begin{array}{l}\mathrm{P} 11 \\
\mathrm{SD} / 3 \mathrm{M}\end{array}$ & $\begin{array}{l}\mathrm{P} 12 \\
\mathrm{SD} / 3 \mathrm{M}\end{array}$ & $\begin{array}{l}\text { Frequency, \% } \\
\text { SD/3M }\end{array}$ \\
\hline
\end{tabular}

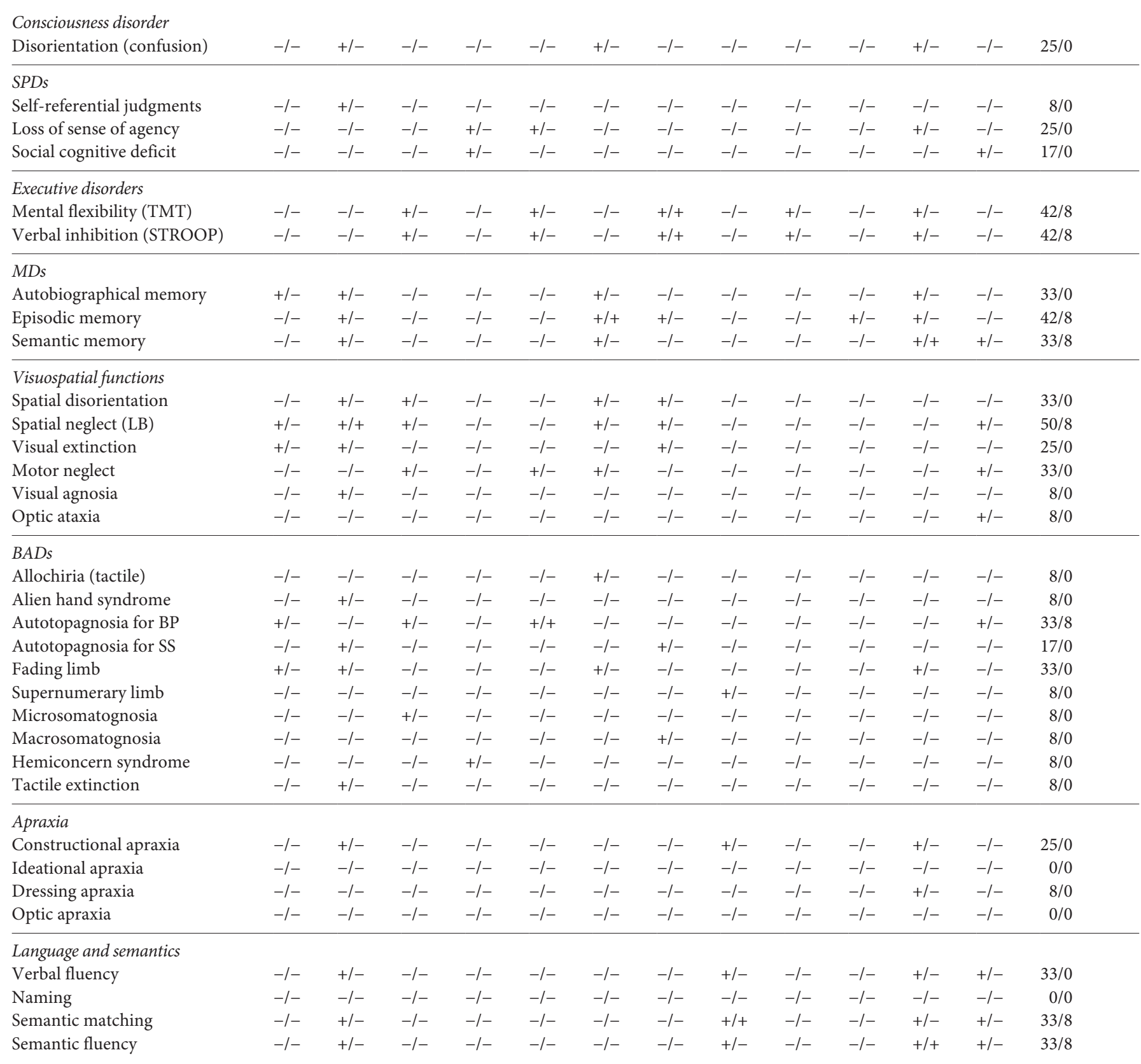

Spatial neglect was present in 7 patients; one of them, P4, had left side lesion. Note that for P1 and P2, the fading limb concerned the entire hemi-body (for P6, it concerned the leg, while for P11, it concerned the hand and the leg). For P9, it concerned the entire hemi-body. For P7, the macrosomatognosia concerned the arm and for P3, microsomatognosia involved the leg and the foot. For P1, 3, and 5, the autotopagnosia for BP involved the entire body part. Semantic matching and fluency deficit were present in the half of patients. +, deficit (i.e., $Z \leq 1.65$ or score < cutoff); -, no deficit; BP, body parts; SS, sensory sensations; SD, first week after stroke; 3M, 3 months after stroke; TMT, Trail Making Test; SPDs, self-processing disorders; BADs, body awareness disorders; MDs, memory disorders. 

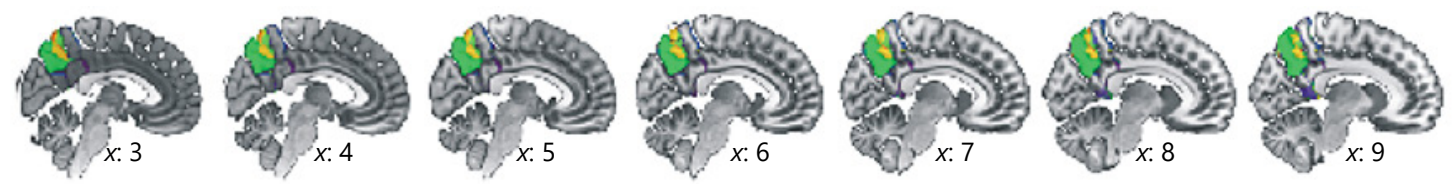

No-SPDs group minus SPDs group
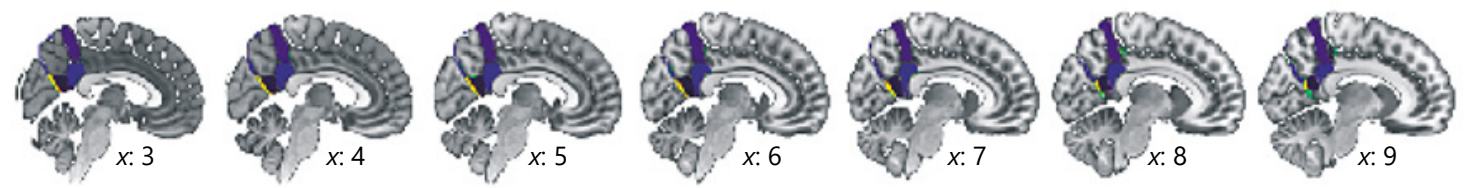

Memory disorders (MDs) group minus no-MDs group
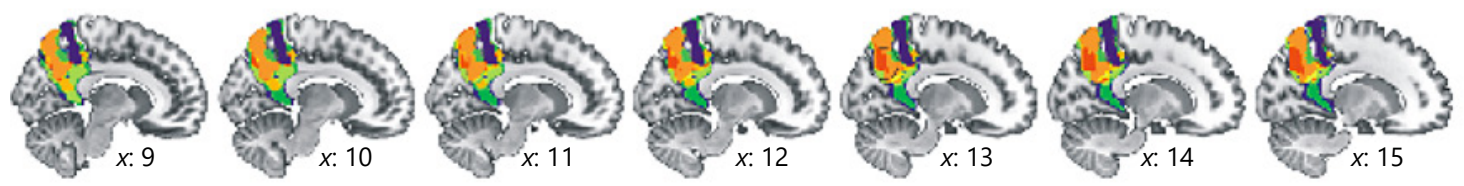

$88 \%(n=7)$

No-MDs group minus MDs group
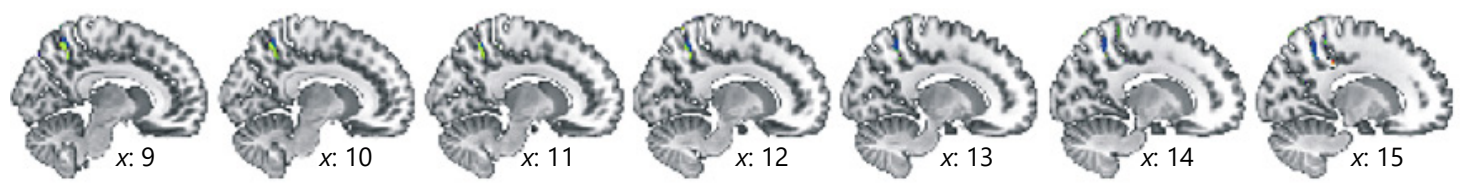

$50 \%(n=2)$

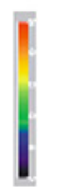

Visuospatial disorders (VSDs) group minus no-VSDs group
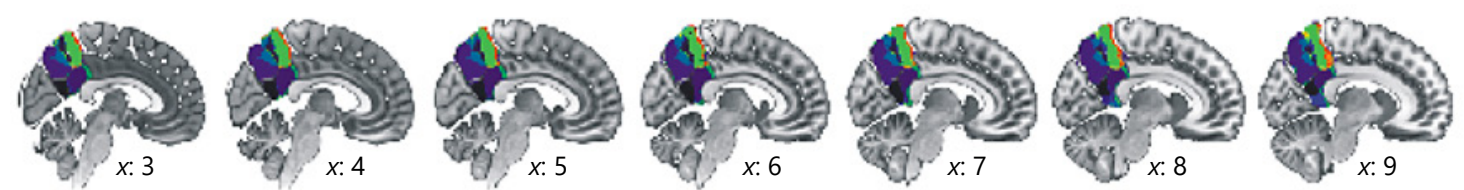

$86 \%(n=6)$
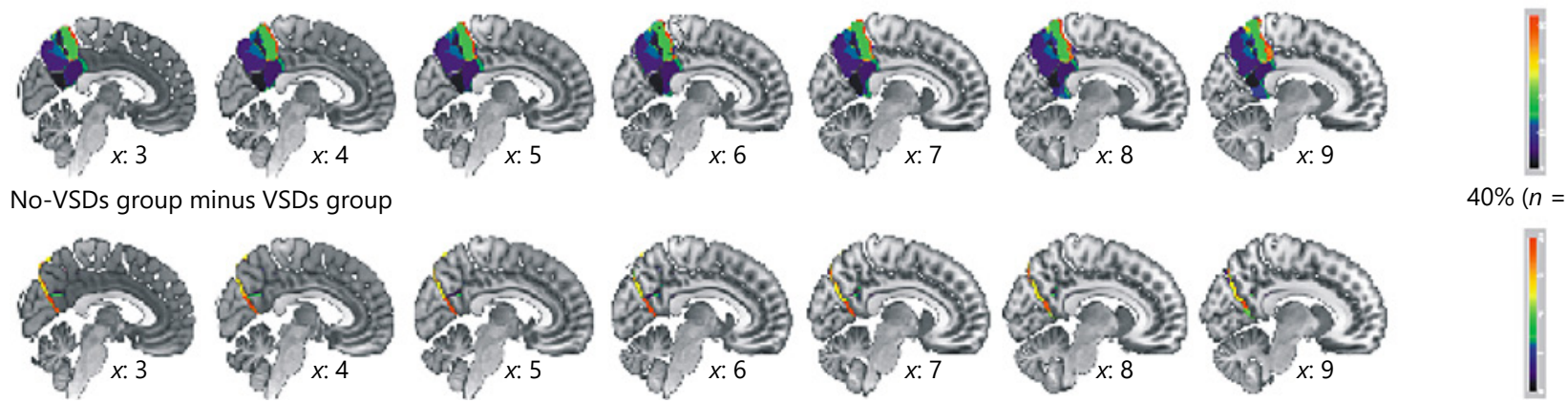

$40 \%(n=2)$

Fig. 3. Subtraction plot results. a The antero-dorsal precuneus was most frequently involved in patients with SPDs compared others (the maximum overlap was $80 \%$ corresponding to $4 / 5$ patients). The postero-dorsal precuneus was most frequently affected in patients with MDs than others (the maximum overlap was $88 \%$ corresponding to $7 / 8$ patients). In patients with VSDs, antero-dorsal precuneus was involved more frequently than others (the maximum overlap was also $86 \%$ corresponding to $6 / 7$ patients). b All

parts of the precuneus involved in BADs (the maximum overlap was $100 \%$ corresponding to $10 / 10$ patients). Antero-dorsal precuneus and posterior splenium were most frequently damaged in patients with semantic processing disorders than others (the maximum overlap was also $75 \%$ corresponding to $3 / 4$ patients). SPDs, self-processing disorders; BADs, body awareness disorders; VSDs, visuospatial disorders; MDs, memory disorders; SePDs, verbal and semantic processing disorders.

(Figure continued on next page.) 


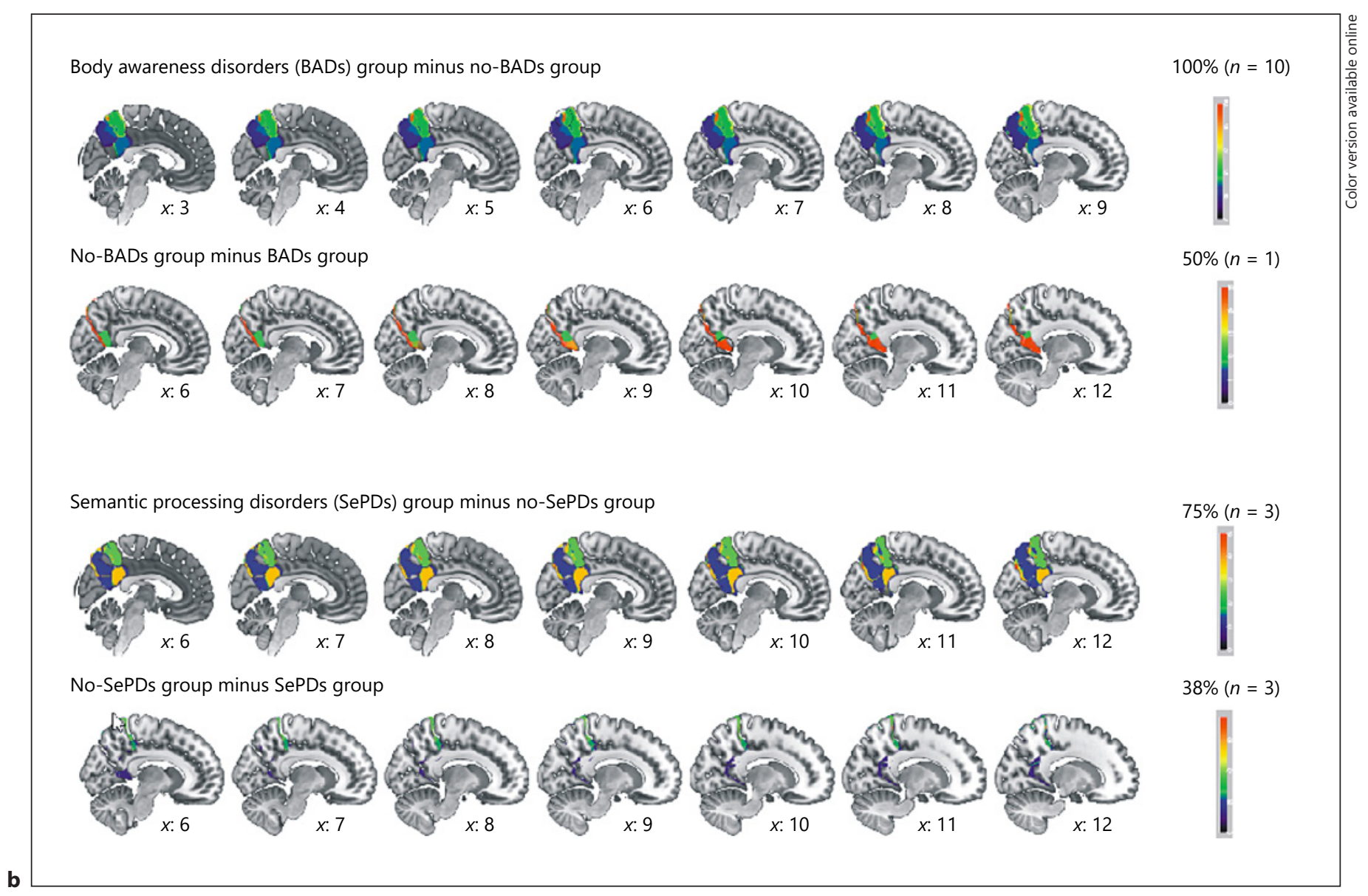

\section{Memory Disorders}

In the acute phase of stroke, autobiographical memory was disturbed in 4 patients (P1, P2, P6, and P11) characterized with loss of important personal events of their life and they could not report self-defining memories. Episodic memory which carries information about personal events and episodes, which are time and space specific, was impaired in 5 patients (P2, P6, P7, P9, and P11). Four patients (P2, P6, P11, and P12) had semantic memory deficits characterized by disturbances of information and knowledge of the world and objects. Patients who showed at least one memory disorder on the first day after stroke $(n=7)$ were more frequently affected by postero-dorsal precuneus damage (maximum $88 \%$ overlap).

\section{Visuospatial Disorders}

Spatial disorientation, characterized by deficits in distinguishing familiar or unfamiliar places, was present in 4 patients (P2, P3, P6, and P7). Spatial neglect demonstrated by line bisection test was found in half of the pa- tients (P2, P3, P6, P7, P11, and P12). The damaged area distribution of cases who showed at least one visuospatial disorder (VSD) after stroke $(n=7)$ contrasted with the damaged area of patients who did not show $\operatorname{VSD}(n=5)$. Patients with VSDs were more often damaged at the level of antero-dorsal precuneus (maximum overlap of $86 \%$ ).

\section{Body Awareness Disorders}

On the first day of stroke, 10 patients had at least one body awareness disorder (BAD; ranging from 1 to 3 ). This contained autotopagnosia for body sensation, fading limb, alien hand, somatoparaphrenia, macrosomatognosia, supernumerary limb, and left/right indistinction. Patients with BADs are presented in Table 3. Fading limb is related to the whole hemi-body for patients $\mathrm{P} 1$ and $\mathrm{P} 2$, the leg for P6, the hand, and the leg for P11. It was characterized by disturbance of "transparency" feeling of their limbs and had difficulty locating the contralesional part of the limbs in the space when they had the eyes closed. 
Three months after stroke, 1 patient continued to experience autotopagnosia for body parts. Patients with BADs $(n=10)$ were damaged at the level of whole precuneus (maximum overlap of 100\%) (Fig. 3b).

\section{Verbal and Semantic Processing Disorders}

Verbal fluency and semantic matching requiring identification of semantically related pictures and names were impaired in 4 patients (P2, P8, P11, and P12). Semantic fluency related to speech monitoring and speech production was disturbed in 3 patients (P2, P11, and P12). On the first day after stroke, the infarction area distribution of patients who showed at least 1 verbal and semantic processing disorder (SePD; $n=4)$ contrasted with the infarction area distribution of patients who did not show SePD $(n=8)$.

\section{Other Neuropsychological Disorders}

Other cognitive disorders were also presented, especially in the poststroke period, including executive disorders (P2, P3, P5, P9, and P11), constructional apraxia (P2, $\mathrm{P} 8$, and $\mathrm{P} 11)$, finger agnosia ( $\mathrm{P} 2$ and $\mathrm{P} 11)$, and optic ataxia (P12). To assess stroke volume in 7 distinct cognitive groups, we used the nonparametric Mann-Whitney test. We found that patients with SPDs, VSDs, BADs, and SePDs had larger stroke volume than patients without $(p<0.05)$.

\section{Discussion}

This study showed the premier evidence for the role of precuneus in various types of cognitive and behavioral functions such as consciousness, orientation, executive functions, spatial and body awareness, and attention. The current study identified that involvement of different areas of the precuneus following ischemic stroke, typically the anterior or the posterior parts, and sometimes the whole precuneus were likely to build the neural basis of these disorders.

In this study, some patients experienced transient confusional state at stroke onset, which was probably caused by impairment of the self and external world integration, this picture of results also suggests that the dysregulation of the network emerging from the precuneus and interconnected posterior cingulate is a possible mechanism [13]. None of the patients had epilepsy or epileptiform activity on EEG. The concurrent proof from recent fMRI studies in healthy individuals suggests that precuneus can play a role in the internal mentation processes of self-

Cognitive Disorders in Precuneal Infract consciousness and self-awareness, by interaction between precuneus and prefrontal cortex $[14,15]$. For instance, patient (P2) with self-referential judgments disorder had an ischemic lesion involving almost all antero-posterior precuneus, while in other SPDs, ischemic lesions concerned a significant part of the precuneus. The interconnected medial prefrontal regions and the anterior precuneus have been suggested to display a network where personal identity and past personal experiences are linked, possibly through the inclusion of mental imaginary strategies, with open interactions that enable us to act between self-representation and awareness [16].

Our topographical analysis indicated that the anterodorsal part of the precuneus was likely to comprise the neural correlate of the VSDs, including spatial disorientation, spatial neglect, visual extinction, and motor neglect. Indeed, fMRI studies have often been reported that the anterior precuneus concerned with execution or preparation of spatially guided behaviors, such as pointing, reaching, and coordinating a complex spatiotemporal motor pattern [17-19]. It is also worth noting that some patients had lesions extending toward the SPL, a border region between anterior and middle cerebral artery. Considering the known functions of these brain regions in spatially guided behavior and cross-modal integration, respectively [20-22], damage to these regions, in principle, can lead to VSDs.

Quite recently, new studies demonstrated the selective role of the medial parietal cortex in autobiographical memory $[23,24]$. The autobiographical memory impairment presented in this study was probably related to the dysfunction of the network between the precuneus, inferior parietal lobule, and posterior cingulate. Moreover, it is worth noticing that some patients experienced episodic and semantic memory disorders which are functionally distinct, though overlapping memory systems might be disturbed by damage of antero-posterior precuneus, whose interlinked selectively with cingulate and prefrontal cortices [25].

It is worth noting that this study showed various type of BADs which are uncommon, following a circumscribed ischemic lesion. It is possible that different aspects of BADs might be developed either by suppression of some tactile information as observed in alien hand and fading limb, and autotopagnosia for body sensations, or by over or underestimation of the size of specific body parts as seen in micro-and macrosomatognosia and supernumerary limbs. This is in agreement with the fact that BADs are related to an impairment of sensorimotor integration [26-31]. Selective breakdown of these pro- 
cesses may lead to a fading limb, as we presented here, 3 of them had a large precuneal infarct, extending toward SPL, experienced a sense of loss of contralesional limb when not under visual control. In fact, integration of both tactile and proprioceptive modalities coming from the contralesional body or maintaining the representation of the current limb position is critical for locating a body member in space [32-34].

It is also important to mention that executive dysfunction continued chronically in a few patients. The disturbances of these processes might be related to the involvement of the posterior cingulate cortex, which was mostly damaged. In fact, the dorsal area of this brain region is linked to frontoparietal networks that provide a continuous activity of highlevel integration between posterior association processes and anterior executive functions $[20,35]$.

This study had some limitations. It should be noted that this study did not perform specific experimental tasks and functional imaging studies. However, this work highlighted that ischemic lesions of the precuneus might cause a variety of cognitive and behavioral disorders, which are related to the disturbance of a broad network of wider associative subcortical and cortical structures. Furthermore, we cannot completely exclude the possibility that ischemic neighboring cortices might be also involved in the development of cognitive deficits. For example, patients P2, P6, and P11 had an infarction involving the paracentral lobule, and in patients $\mathrm{P} 5$ and $\mathrm{P} 11$, the ischemic lesion also involved a large part of the SPL. Given the established role of these brain areas in self-processing, visuospatial functions, somatosensory processing, ischemic damage to these regions might, as a result, lead to a wide spectrum of cognitive impairment. It is worth noting that in some cases, the posterior cingulate gyrus was also affected, which has reached connections with sensorimotor regions [20] and fronto-parietal networks involved in cognitive control. Some ischemic lesions extended laterally into the SPL, which is known to play a role in visuospatial information processing $[1,21,22]$. Eventually, it seems that a lesion involving these structures may cause distinct but related cognitive disorders. Another limitation of our study is that MR images taken 3 months after stroke were not analyzed and the evaluations in that period were not available.

The main cause of precuneal ischemic lesions was embolism, which is originated from atrial cardiopathy and carotid atherosclerotic plaques. Isolated and circumscribed lesions in the precuneus could most likely be due to the fact that these small parts of embolism damaged certain small areas.
As a result of all these data, this study corroborates that the precuneus plays a wide variety of potential functions, highly specialized that can no longer be seen as a simple extension of the sensory motor and visuospatial processes supported by the neighboring cortices. This study clarifies that more studies are needed to consistently address the role of the precuneus in relation to its activity with the specific part of the neural network.

\section{Acknowledgements}

We are grateful to all the patients who took part in the study. We would especially like to thank the clinical staff at the Department of Neurology, Ege University, School of Medicine, İzmir Türkiye. We would like to acknowledge the contribution of $\mathrm{Mr}$ Hasan Arslan and Ms Günay Çoşkun toward analysis of the neurocognitive tests.

\section{Statement of Ethics}

This study complied with the guidelines for human studies and includes evidence that the research was conducted ethically in accordance with the World Medical Association Declaration of Helsinki. Ege University Medical Ethical Committee approved this study following the principles outlined in the Helsinki Declaration before starting the study (No. 2013/50). The authors declare that subjects (or their parents or guardians) have given their written informed consent.

\section{Conflict of Interest Statement}

We have no actual or potential conflicts of interest for all authors involved in this paper.

\section{Funding Sources}

Emre Kumral, MD, Fatma Ece Bayam, MD, and Hüseyin Nezih Özdemir, MD did not receive funding disclosure.

\section{Author Contributions}

Principal author: Emre Kumral, MD. Study concept or design: Emre Kumral, MD and Fatma Ece Bayam, MD. Acquisition of data: Hüseyin Nezih Özdemir, MD. Analysis or interpretation of data: Hüseyin Nezih Özdemir, MD. Statistical analysis: Emre Kumral, MD. Study supervision or coordination: Emre Kumral, $\mathrm{MD}$ and Fatma Ece Bayam, MD. 


\section{References}

1 Cavada C, Goldman-Rakic PS. Posterior parietal cortex in rhesus monkey: II. Evidence for segregated corticocortical networks linking sensory and limbic areas with the frontal lobe. J Comp Neurol. 1989;287(4):422-45.

2 Berlucchi G, Aglioti S. The body in the brain: neural bases of corporeal awareness. Trends Neurosci. 1997;20(12):560-4.

3 Margulies DS, Vincent JL, Kelly C, Lohmann G, Uddin LQ, Biswal BB, et al. Precuneus shares intrinsic functional architecture in humans and monkeys. Proc Natl Acad Sci U S A. 2009;106(47):20069-74.

4 Zhang S, Li CS. Functional connectivity mapping of the human precuneus by resting state fMRI. Neuroimage. 2012;59(4):3548-62.

5 Maravita A, Spence C, Driver J. Multisensory integration and the body schema: close to hand and within reach. Curr Biol. 2003; 13(13):R531-9.

6 Dijkerman HC, De Haan EH. Somatosensory processing subserving perception and action: dissociations, interactions, and integration. Behav Brain Sci. 2007;30:224-30.

7 Cavanna AE, Trimble MR. The precuneus: a review of its functional anatomy and behavioural correlates. Brain. 2006;129(Pt 3):56483.

8 Karnath HO, Perenin MT. Cortical control of visually guided reaching: evidence from patients with optic ataxia. Cereb Cortex. 2005; 15(10):1561-9.

9 Boes AD, Prasad S, Liu H, Liu Q, Pascual-Leone A, Caviness VS Jr, et al. Network localization of neurological symptoms from focal brain lesions. Brain. 2015;138(Pt 10):306175.

10 Herbet G, Lemaitre AL, Moritz-Gasser S, Cochereau J, Duffau H. The antero-dorsal precuneal cortex supports specific aspects of bodily awareness. Brain. 2019;142(8):220714.

11 Kumral E, Ozkaya B, Sagduyu A, Sirin H, Vardarli E, Pehlivan M. The ege stroke registry: a hospital-based study in the Aegean Region, Izmir, Turkey. Cerebrovasc Dis. 1998;8: 278-88

12 Adams HP Jr, Bendixen BH, Kappelle LJ, Biller J, Love BB, Gordon DL, et al. Classification of subtype of acute ischemic stroke: definitions for use in a multicenter clinical trial: TOAST Trial of Org 10172 in acute stroke treatment. Stroke. 1993;24:35-41.
13 Laureys S, Goldman S, Phillips C, Van Bogaert $\mathrm{P}$, Aerts J, Luxen A, et al. Impaired effective cortical connectivity in vegetative state: preliminary investigation using PET. Neuroimage. 1999;9(4):377-82.

14 Kircher TTJ, Senior C, Phillips ML, Benson PJ, Bullmore ET, Brammer M, et al. Towards a functional neuroanatomy of self-processing: effects of faces and words. Cogn Brain Res. 2000;10:133-44.

15 Kjaer TW, Nowak M, Lou HC. Reflective selfawareness and conscious states: PET evidence for a common midline parietofrontal core. Neuroimage. 2002;17(2):1080-6.

16 Andreasen NC, O'Leary DS, Cizadlo T, Arndt S, Rezai K, Watkins GL, et al. Remembering the past: two facets of episodic memory explored with positron emission tomography. Am J Psychiatry. 1995;152(11):1576-85

17 Gitelman DR, Nobre AC, Parrish TB, LaBar KS, Kim YH, Meyer JR, et al. A large-scale distributed network for covert spatial attention: further anatomical delineation based on stringent behavioural and cognitive controls. Brain. 1999;122(Pt 6):1093-106.

18 Beauchamp MS, Petit L, Ellmore TM, Ingeholm J, Haxby JV. A parametric fMRI study of overt and covert shifts of visuospatial attention. Neuroimage. 2001;14(2):310-21

19 Suchan B, Yágüez L, Wunderlich G, Canavan AG, Herzog H, Tellmann L, et al. Hemispheric dissociation of visual-pattern processing and visual rotation. Behav Brain Res. 2002; 136(2):533-44.

20 Leech R, Kamourieh S, Beckmann CF, Sharp DJ. Fractioning the default mode network: distinct contributions of the ventral and dorsal posterior cingulate cortex to cognitive control. J Neurosci. 2011;31:3217-24.

21 Selemon LD, Goldman-Rakic PS. Common cortical and subcortical targets of the dorsolateral prefrontal and posterior parietal cortices in the rhesus monkey: evidence for a distributed neural network subserving spatially guided behavior. J Neurosci. 1988;8(11):4049-68.

22 Leichnetz GR, Goldberg ME. Higher centers concerned with eye movement and visual attention: cerebral cortex and thalamus. In: Buttner-Ennever JA, editor. Neuroanatomy of the oculomotor system. Amsterdam: Elsevier; 1988. Vol. 2; p. 365-429.
23 Addis DR, McIntosh AR, Moscovitch M, Crawley AP, McAndrews MP. Characterizing spatial and temporal features of autobiographical memory retrieval networks: a partial least squares approach. Neuroimage. 2004;23(4):1460-71.

24 Gilboa A, Winocur G, Grady CL, Hevenor SJ, Moscovitch M. Remembering our past: functional neuroanatomy of recollection of recent and very remote personal events. Cereb Cortex. 2004;14(11):1214-25.

25 Wheeler MA. Episodic memory and autonoetic awareness. In: Tulving E, Craik FIM, editors. The Oxford handbook of memory. Oxford: Oxford University Press; 2000. p. $597-$ 608

26 Wolpert DM, Goodbody SJ, Husain M. Maintaining internal representations: the role of the human superior parietal lobe. Nat Neurosci. 1998;1(6):529.

27 Feinberg TE, Haber LD, Leeds NE. Verbal asomatognosia. Neurology. 1990;40(9):1391.

28 Karnath HO, Baier B, Nägele T. Awareness of the functioning of one's own limbs mediated by the insular cortex? J Neurosci. 2005;25(31): 7134-8.

29 Feinberg TE, Venneri A, Simone AM, Fan Y, Northoff G. The neuroanatomy of asomatognosia and somatoparaphrenia. J Neurol Neurosurg Psychiatry. 2010;81(3):276-81.

30 Weijers NR, Rietveld A, Meijer A, De Leeuw FE. Macrosomatognosia in frontal lobe infarct: a case report. J Neurol. 2013;260:925.

31 Vallar G, Ronchi R. Somatoparaphrenia: a body delusion. A review of the europsychological literature. Exp Brain Res. 2009;192: 533-51.

32 Vuilleumier P, Reverdin A, Landis T. Four legs: illusory reduplication of the lower limbs after bilateral parietal lobe damage. Arch Neurol. 1997;54:1543-7.

33 Graziano MS. Where is my arm? The relative role of vision and proprioception in the neuronal representation of limb position. Proc Natl Acad Sci U S A. 1999;96(18):10418-21.

34 Holmes NP, Spence C. The body schema and multisensory representation(s) of peripersonal space. Cogn Process. 2004;5:94-105.

35 Leech R, Sharp DJ. The role of the posterior cingulate cortex in cognition and disease. Brain. 2014;137(Pt 1):12-32. 